\title{
A Dynamic Channel Allocation Algorithm in TD-SCDMA System
}

\author{
Hongyan $\mathrm{Hu}^{1}$, Zhenhong $\mathrm{Jia}^{2} *$, Xizhong Qin ${ }^{3}$, Chuanling $\mathrm{Cao}^{4}$ and Hongmei $\mathrm{Niu}^{5}$ \\ 1,2,3 Xinjiang University \\ ${ }^{4,5}$ China Mobile group Xinjiang Co., Ltd. \\ ${ }^{1} 15894694711 @ 163 . c o m,{ }^{2} j z h h @ x j u . e d u . c n$
}

\begin{abstract}
In the TD-SCDMA system, there is a problem that non-emergency voice services probability of dropped call is not ideal when we use the dynamic allocation algorithm. To solve this problem, we propose a new algorithm, which is called protect channel reservation queue movable boundary dynamic channel allocation. This algorithm set aside channels for dropped calls of non-emergency voice services service, in order to keep blocking probability for service, the introduction of no patient customers, combined with the data packet to borrow algorithms. Simulation results show that, comparing to the movable boundary algorithm, the proposed algorithm reduce the drop probability for non-emergency voice services, and maintain a variety of service blocking probability, reducing the packet loss probability data services.
\end{abstract}

Keywords: TD-SCDMA; protect channel relieve queue movable boundary dynamic channel allocation; impatient customers; channel reservation

\section{Introduction}

TD-SCDMA mobile communication system is the first generation system of telecommunications technology in China which has independent intellectual property rights. In TD-SCDMA system, many key technologies are used, including duplex time division, smart antennas, joint detection and dynamic channel allocation, etc. Among these technologies, the dynamic channel allocation played an important role in reducing interference, increasing system capacity and improving the quality of service, so that the limited sources can well serve the growing number of user. According to the 3GPP standard, DCA can be divided into slow dynamic channel allocation (SDCA) and fast dynamic channel allocation (FDCA). SDCA refers to allocate resources to cells in TD-SCDMA system, while FDCA is allocate resources for the user of application access, and according to the system status the resources to be adjusted, which have been allocated.

At present, for TD-SCDMA system the study of fast dynamic channel allocation mainly concentrated in the high-priority voice service, mobile boundary algorithm, handoff call priority channel allocation strategies, cross-slot allocation algorithm based fast dynamic channel interference, etc. [1-5, 11]. There are few researches about the TD-SCDMA system in other country, because the TD-SCDMA system was originally raised in China, and the research about the allocation of channel in this system is still not deep. Nowadays, more researches are done on channel allocation method of movable boundary strategy. This method is from M.J.Fisher et. Who in the future put forward the strategy of mobile border dynamic channel allocation (MB_DCA) which is suitable to wireless communication system applications [12-13]. In this strategy, all time slots are divided into two parts which are data service time slots and voice service time slots. When service transfer is processing, if the voice service time slots are idle, data service data 
packets in the buffer queue can be transmitted by borrowing the voice service time slots. But when a voice call comes in, the voice service can seize the voice time slots borrowed by data services for voice communications. Then a hybrid data rate, small grouping borrowed MB_DCA algorithm (MRG_MB_DCA) has been proposed [6], compared with the original MB_DCA, on the base of not affecting the probability of call blacking, this algorithm reduces the probability of data packet loss and improves the system resources utilization, but the article has not considered the voice service priority; based on MRG_MB_DCA channel reserved for handoff calls, the PCR_MB_DCA has been proposed [7], the algorithm reduces the blocking probability of voice handoff calls and improves the performance of data services; queue movable boundary dynamic channel allocation (QMB_DCA) has been proposed [8],it reduces probability of blocking and probability of dropped call in low priority service, but the probability of dropped call in high priority service does not improve.

To solve these problems, a protect channel relieve queue movable boundary dynamic channel allocation (PCR_QMB_DCA) is proposed, the algorithm improves the queue movable boundary dynamic channel allocation (QMB_DCA). PCR_QMB_DCA algorithms reserved channels for dropped call service, which is non-emergency voice dropped call service, thereby reducing the probability of dropped call for voice calls; Since PCR_QMB_DCA introduced the no patience customer, so that ensures probability of blocking unchanged on the basis of lower drop probability; taking into account the different service level of data service have different probability, reduce the packet loss probability.

\section{TD-SCDMA System Frame Structure}

TD-SCDMA system, each RF channel bandwidth of $1.6 \mathrm{MHz}$, chip probability $1.28 \mathrm{Mchip} / \mathrm{s}$. TD-SCDMA system radio frame period is $10 \mathrm{~ms}$, each radio frame is divided into two 5ms sub- frames and each sub-frame is then subdivided into 3 special time slots and 7 time slots. As shown in Figure 1. In seven regular time slots, TS0 is always allocated as the downlink direction and as a broadcast communication channel, TS1-TS6 slots for service, carrying uplink and downlink service, there is switching point that separate the uplink and the downlink. 3 special time slots are uplink pilot time slot (UpPTS), the guard period (GP) and downlink pilot time slot (DwPTS), the guard period is to provide protection for the uplink and downlink data transmission, as shown in Figure $1[10]$. 


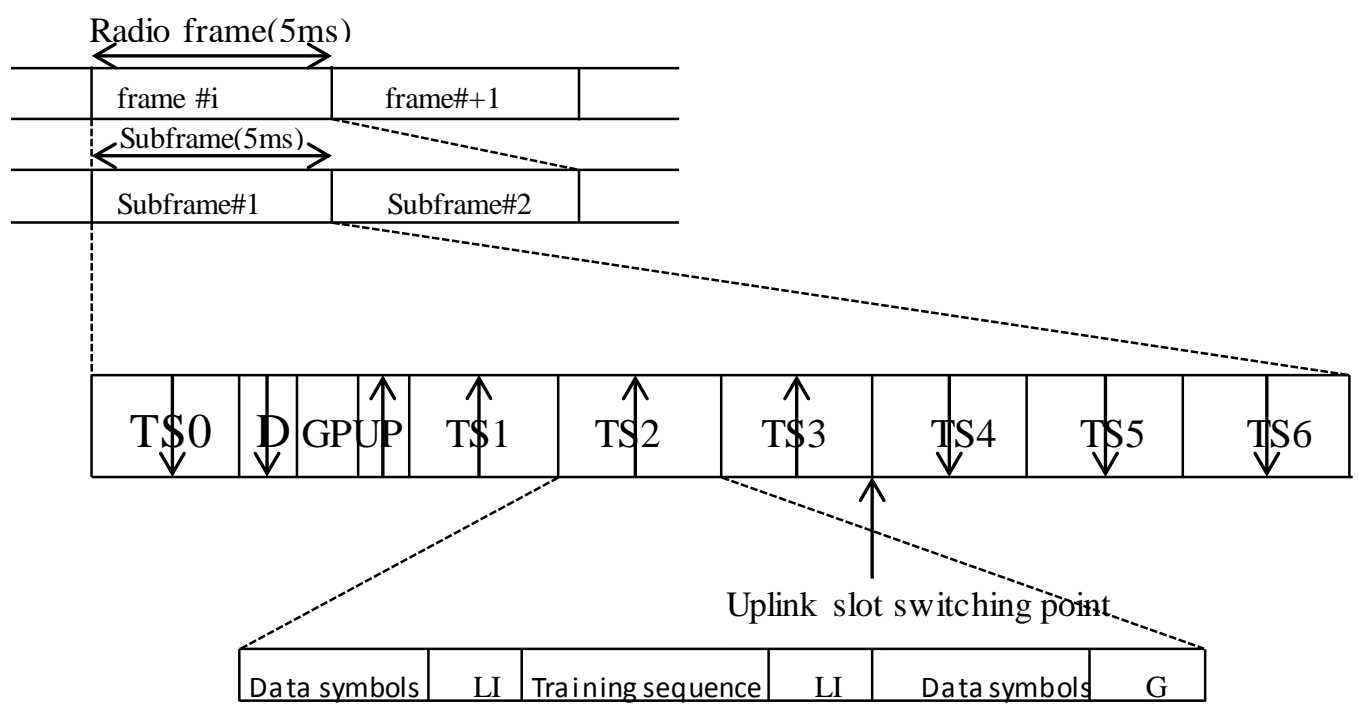

Figure 1. The Frame Structure of TD-SCDMA

In TD-SCDMA system, one of service time slots can be divided into 1, 2, 4, 8, 16 code channels according to spreading factor. In this paper the spreading factor (SF) is 16, a channel as a basic resource unit (BRU), so that 16 basic resource units in a slot. For the different service classes of different service the transmission rate is different and BRU desired different. The rate of voice service is $12.2 \mathrm{kbps}$, required $2 \mathrm{BRU}$, typical data transferring rate of flow data service are asked to $64 \mathrm{kbps}$, which need $7 \mathrm{BRU}$, while data transferring rate of interaction class service is $144 \mathrm{kbps}$ needed $16 \mathrm{BRU}$ [9].

\section{Protect Channel Reservation Queue Movable Boundary Dynamic Channel Allocation (PCR_QMB_DCA)}

\subsection{Queue Movable Boundary Dynamic Channel Allocation Algorithms}

In queue movable boundary dynamic channel allocation(QMB_DCA) algorithms, the voice service is divided into emergency service, preemption priority voice service and ordinary voice service. Among them, the emergency service are the most important service in trunking system, In the service transfer processing, if there is no idle channel available, the emergency services can seize channels, which each other non-urgent priority service channel. For preemption priority call, depending on the setting system can seize channels, which is lower-priority ordinary trunking service channel if there is no idle channel is available. If the voice channels are idle and data services buffer queue is not empty, data packets in the buffer queue can transmit by borrowing the voice channels. Befor borrow the channels, check up whether there are 16 or more than 16 voice time slots BRU idle in the first, if so, the first packet in the register will be transmitted by borrowing voice service slots; if the idle voice time slots BRU less 16, but greater than 8 , the $64 \mathrm{kbit} / \mathrm{s}$ data service will be transmitted by borrowing voice service slots. If less than 8 BRU continue to wait in the queue; When a voice call comes in, voice services can seize the voice channels borrowed by data services for voice communications and data 
service continue to wait in line, QMB_DCA model as shown in Figure 2 [8-9]:

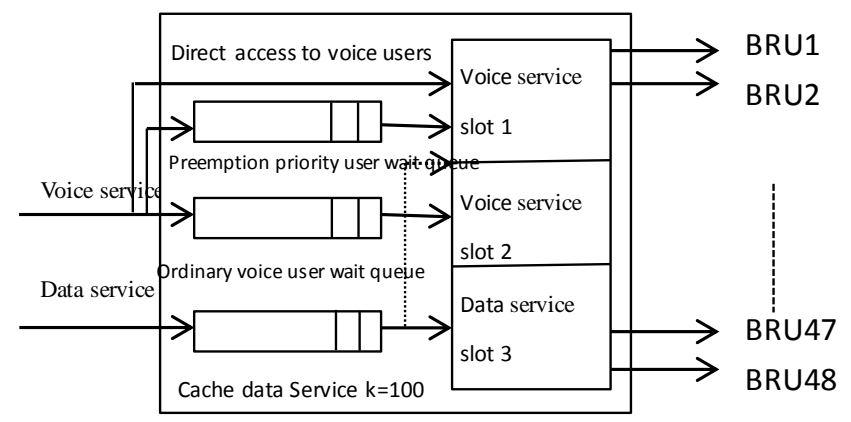

Figure 2. QMB_DCA Model

QMB_DCA algorithm considers the priority of voice services, reducing the probability of blocking and probability of dropped call for low-priority services, but the QMB_DCA does not improve probability of dropped call than MB_DCA algorithm for preemption priority services, and the probability of dropped call for ordinary voice service is not ideal, it does not consider the different rate data services need different BRU. In order to make better use of system resources, reducing call drop probability and improving the performance of data service. A protect channel relieve queue movable boundary dynamic channel allocation algorithms is proposed which is reserved channel for non-emergency voice, introducting no patient customers, combining group borrowing strategy with data service.

\subsection{The Design Ideas of Channel Reservation Movable Boundary Algorithm}

Taking into account the service blocking will not only bring negative impact on the performance of the cell, while the call abruptly leaving the user annoyed, so if keep a low blocking probability in voice calls and reduce the probability of dropped calls, then can increase quality of service (QOS). In reality, when the voice call comes in, the users will often hang up for a while if the system does not have available channels. During the waiting time if a serving call goes away, the new call can get the free channels to complete access, if waiting for a while is still no channel available then to leave. So queue policies can effectively reduce the voice services probability of blocking, on this basis, consider if it is some channels is specialized reserved for dropped calls service which is non-priority voice service dropped calls, thus reducing non-priority service probability of dropped call. However, due to the reserved channel will affect the probability of blocking, considering the introduction of no patient customers, just as some customers queuing process because there is no patience and left, so that you can keep the original blocking probability.

In PCR_QMB_DCA algorithm, followed QMB_DCA algorithm will be divided into three levels of voice service. Reserved channel for non-emergency voice service call drop, preemption priority service for the right to use the channel is reserved than ordinary service. The reserved channel is to be used by the normal services when preemption priority service's dropped call isn't exist, at the beginning of. If the dropped call probability of preemption priority service is lower than $5 \times 10^{-5}$, the ordinary services allocated to use the channel, when preemption priority service having dropped calls. For data services, we will division two groups in each time slot when the data services buffer queue is not empty and need to borrow idle channels of voice service, each group has 8 $\mathrm{BRU}$, so the total number of the voice time slots group is 4 . Befor borrow the channels, 
check up how much groups idle channels there are. if the idle voice time slots group is 1 the $64 \mathrm{kbit} / \mathrm{s}$ data service will be transmitted by borrowing voice service slots. If has 2 the $144 \mathrm{kbit} / \mathrm{s}$ browser class data service or two-way $64 \mathrm{kbit} / \mathrm{s}$ streaming class data service will be transmitted by borrowing voice service slots. But if these idle BRU which scatter in the different time slots are fragmented and not enough resources can meet the data service in the same time slot. In this case, these scattered idle BRU need to adjust into a time slot for meet the need of data service access. And the data service can borrowe reserved channels. The system model as shown in Figure 3:

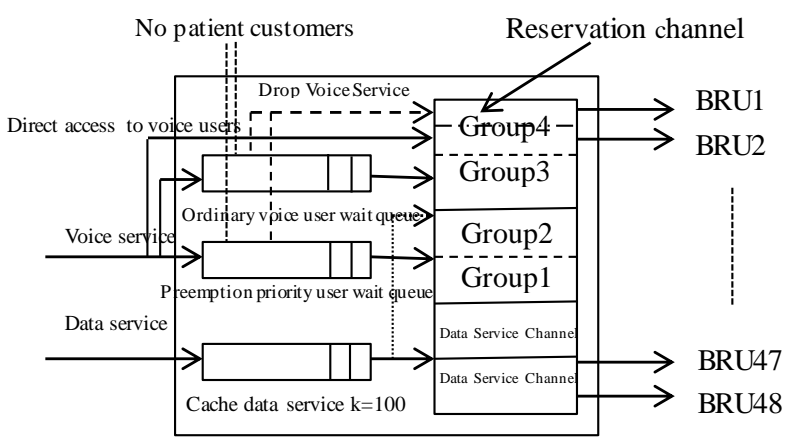

Figure 3. PCR_QMB_DCA Model

The essence of the PCR_QMB_DCA is compulsive interruption during a call, which is more unbearable than the short waiting time. In order to lower the probability of dropped call in voice service, reserve certain channels for dropped call service that may be non-emergency, when the probability of dropped call service whose priority is higher or captures is lower than the definite value, ordinary services of voice are able to share the reserved channels temporarily. In order to avoid the problem that the probability of blocking of the other service increases because of the channel reservation, we introduce no patient customers, who flow away by Poisson distribution, and the departing strength of the customers is directly proportional to the length of the queue. And in the line of voice service, we postponing the access of the non-emergency services in order to make the rate of blocking in a low level. It can enhance the utilization of the channel that we add no patient customers in the combination of the queuing theroy and reservation of channel. For non-real-time data services the length of the register is limited, packet first enters a FIFO register until obtaining the necessary channel, but if the register is full, dropping the data packet will occur.

\subsection{Performance Analysis}

According to the service model in TD-SCDMA system, the arriving probability of voice calls and data packets belong to Poisson distribution, the duration of voice calls and data packet transmission time all meet negative exponential distribution, and the arrival process independent. Non-patient customers left queue by Poisson distribution, set the leave strength of no patient customers for preemption priority service is $\beta_{j}$, leaveing strength of non-patient customers for normal service is $\alpha_{i}$; Its queue lengths are $j$ and $i, \beta_{\mathrm{j}}$ $=\gamma j(\gamma>0), \alpha_{i}=\lambda i(\lambda>0)$ 。 Preemption priority services and ordinary voice services were set FIFO buffer queue, set timeout for Tv1 and Tv2, and there Tv2> Tv1. 


\subsubsection{New User Access}

When a new call arrives, according to the following procedure for channel allocation, as shown in Figure 4:

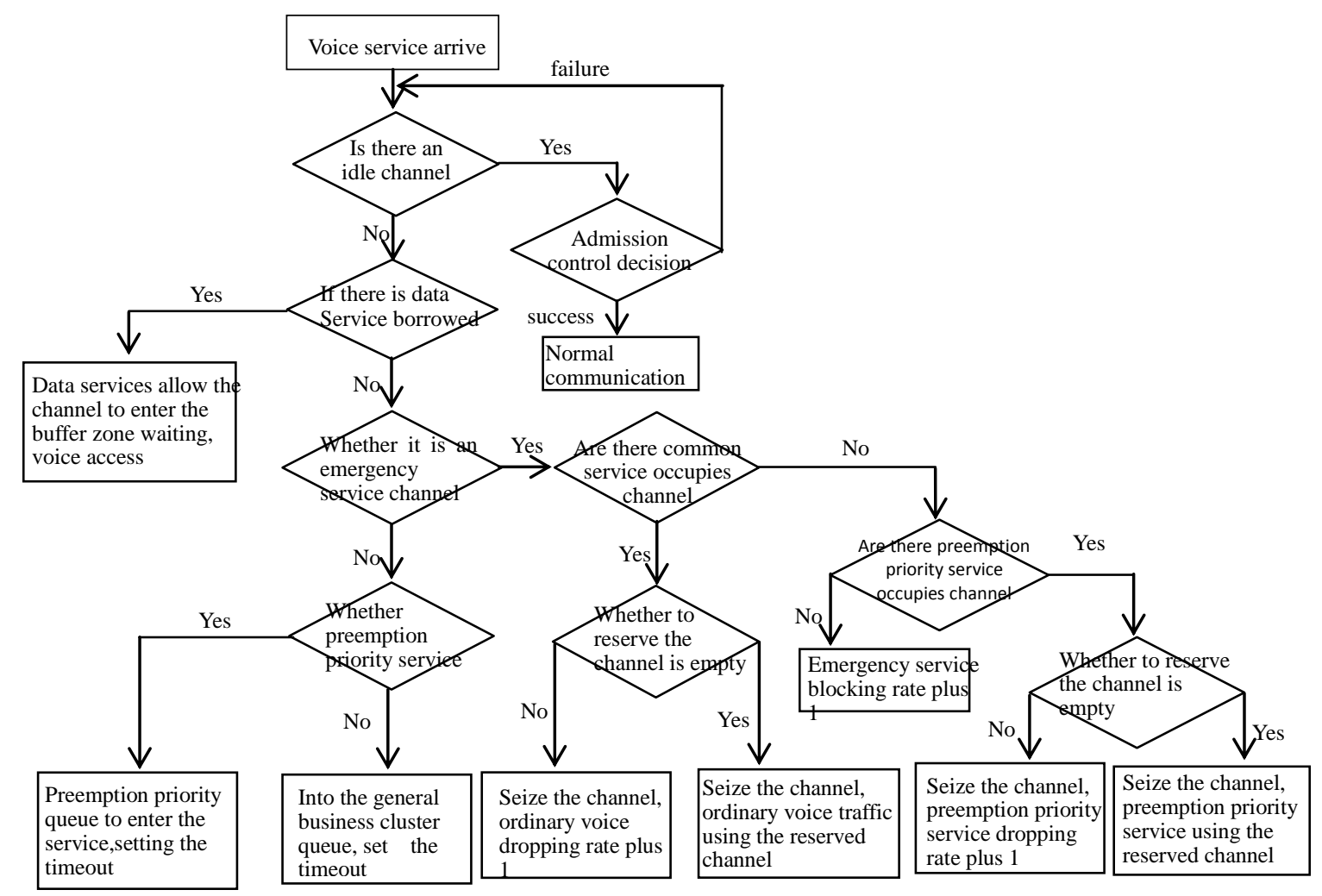

Figure 4. Channel Assignment Flow Chart of New Service Reaches

\subsubsection{Loop Detection}

The system loop detection when no new call comes in. The first service in the high-priority voice service queue will be transmitted when there is an idle voice channel, if the high-priority voice service queue is empty the first service in the low-priority voice service queue will be transmitted, and if the low-priority voice service queue is empty then examine whether voice slots have enough idle channels, if so, the first packet in the register will be transmitted by borrowing voice service slots; When the idle channel is zero, check up whether the timeout service is exist in the first, if there is timeout service in the high-priority voice service queue and then examine whether the voice channel be borrowed by data service, if so, voice timeout service can seize the voice channels borrowed by data services for voice communications and data service continue to wait in line; if there is no voice channel be borrowed by data service that voice timeout service can seize the voice channels by normal services for voice communications, and then check up whether the reserved channel is empty, if so, it be used by dropped call of normal service; if not for, the normal service dropping probability plus 1 ; if neither data services nor ordinary voice services occupy the voice channel, timeout service will be removed from the queue, preemption priority service blocking probability plus 1 . If there is a timeout service in the ordinary voice services queue, examine whether the voice channel be borrowed by data service, if so, normal voice timeout service can seize the voice channels borrowed by data services for voice communications and data service 
continue to wait in line, if not for, timeout service will be removed from the queue and ordinary service blocking probability plus 1 .

\section{Simulation Results and Analysis}

In order to verify the performance of the proposed algorithm, we did an emulation analysis between the proposed algorithm (PCR_QMB_DCA) and the QMB_DCA and MB_DCA algorithm in literature [9]. The analysis was carried out in terms of emergency voice services and normal voice services of dropped calls and blocking probability, and packet loss probability of data services, probability of channel utilization. The arriving probability of voice calls and data packets obey Poisson distribution, and the service time obeys negative exponential distribution. The main simulation parameters are presented in Table 1.

Table 1. Simulation Parameters

\begin{tabular}{|l|l|}
\hline Parameters & Value \\
\hline a subframe Service number of slots & 6 \\
\hline Up / down ratio slots & $3: 3$ \\
\hline Each slot number BRU & 16 \\
\hline Voice call arrival distribution & Poisson distribution \\
\hline Voice duration of the call distribution & Negative exponential distribution \\
\hline Rate of voice service & $12.2 \mathrm{kbit} / \mathrm{s}$ \\
\hline Required BRU for voice services & 2 \\
\hline Voice call arrival probability & $0 \sim 600$ (call/h) \\
\hline Data packet arrival distribution & Poisson distribution \\
\hline $\begin{array}{l}\text { Distribution of data packet transmission } \\
\text { time }\end{array}$ & Negative exponential distribution \\
\hline Rate of data service & $64 \mathrm{kbit} / \mathrm{s}$ 、 144kbit/s \\
\hline Required BRU for data services & BRU 8 、16 \\
\hline Data buffer length & 100 \\
\hline Reserved number of channels & 1 \\
\hline
\end{tabular}

According to Table 1, the time slot ratio of uplink and downlinkis 3: 3, and the time slot ratio of voice and data service is $2: 1$, and arrival probability ratio of the emergency service, preemptive priority service and normal voice service is $1: 4: 5$. Based on the algorithm simulation matlab platform, the simulation results are showed in Figure 4-9. 


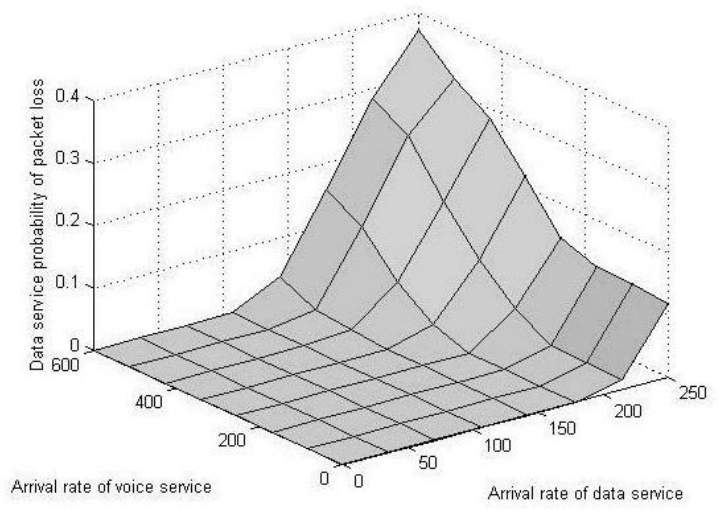

Figure 5. QMB_DCA Data Service Probability of Packet Loss

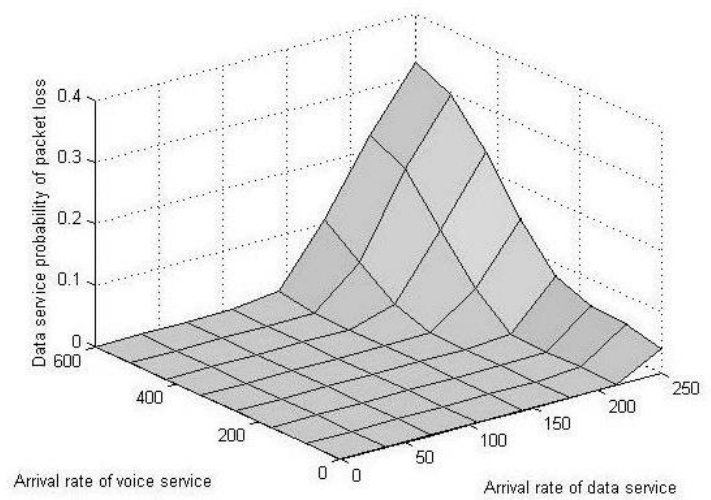

Figure 6. PCR_QMB_DCA Data Service Probability of Packet Loss

According to Figure 5 and Figure 6, we can see the packet loss probability for data services effectively reduced in the PCR_QMB-DCA algorithms compared to the QMB_DCA. The reason is that the improved algorithms adopt grouping borrowing stprobabilitygy which increase the probability of data services borrow voice channel.

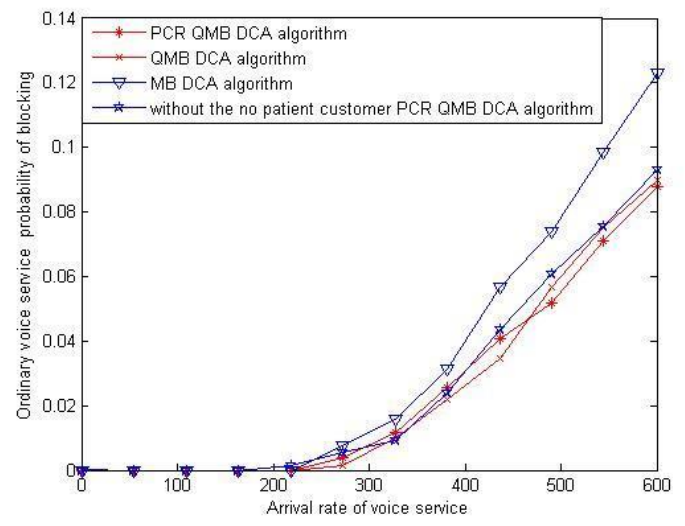

Figure 7. Three Algorithms Ordinary Voice Services Probability of Blocking 


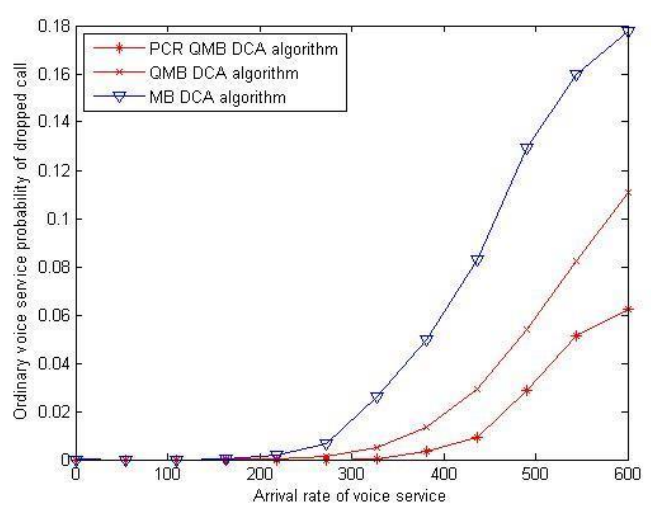

Figure 8. Three Algorithms Ordinary Voice Service Probability of Dropped Call

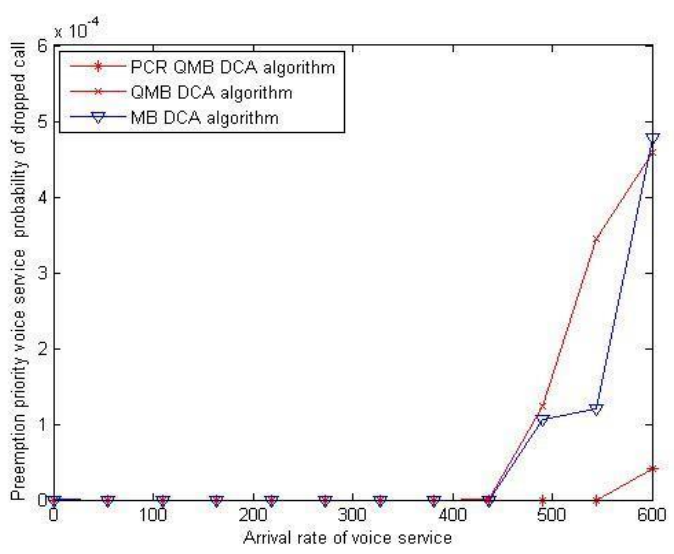

Figure 9. Three Algorithms Preemption Priority Voice Service Probability of Dropped Call

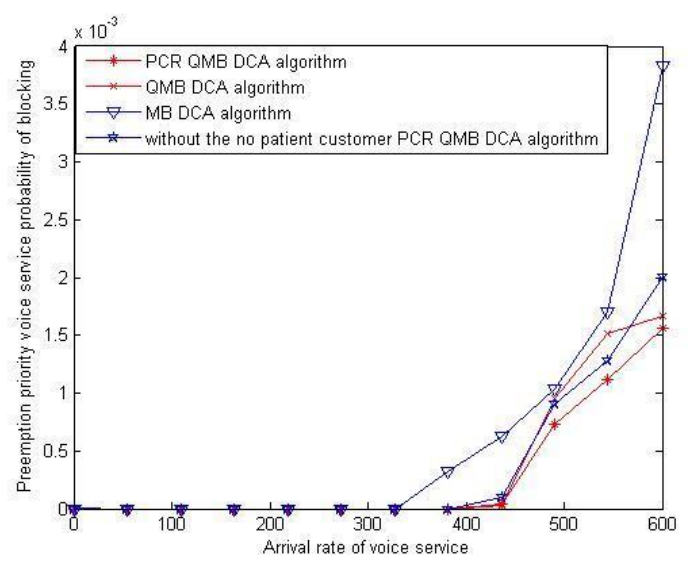

Figure 10. Three Algorithms Preemption Priority Voice Services Probability of Blocking 


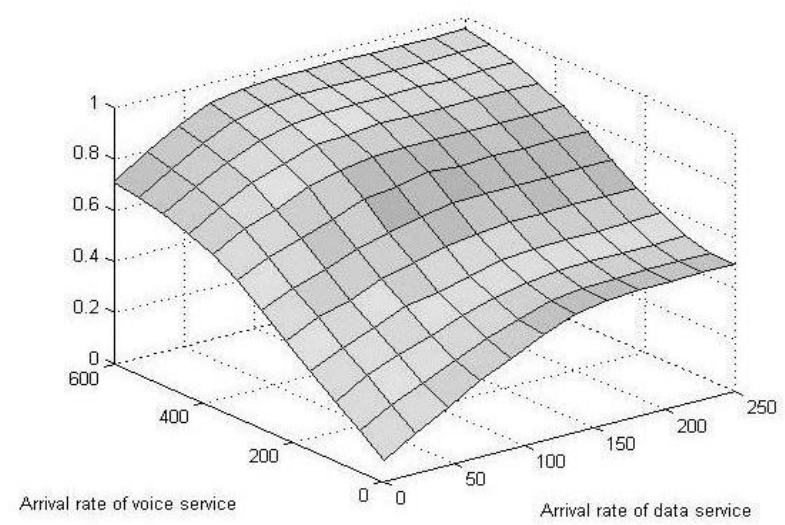

Figure 11. QMB_DCA Probability of Channel Utilization

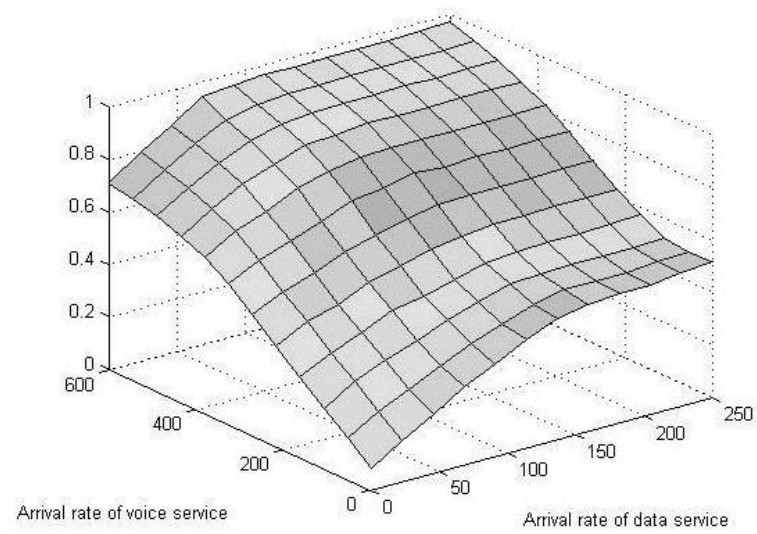

Figure 12. PCR_QMB_DCA Probability of Channel Utilization

It can be seen from Figure 7 and 10 that the blocking probability for non-emergency voice services in PCR_QMB-DCA algorithms which is without non-patient customer is slightly higher than it is in PCR_QMB_DCA and MB_DCA algorithms, while the blocking probability for non-emergency voice services in the PCR_QMB_DCA and QMB_DCA algorithms remain the same and is lower than it is in MB_DCA algorithms. For MB_DCA algorithms the voice service is real-time service, and if it do not get the needed channel the call will be blocked, both PCR_QMB_DCA and QMB_DCA algorithms adopt a queuing strategy in which calls enter a FIFO register firstly until the setted waiting time is exceeded, so the blocking probability reducesed greatly than it is in MB_DCA algorithms.

According to settings the reserved channel is to be used by the ordinary services when the dropped call probability of preemptiong priority service is lowwer than $5 \times 10^{-5}$. It can be seen from Figure 8 and 9 that the dropped call probability for ordinary service and preemption priority service are reduced. From Figure 9 we can see the preemption priority service of dropped call is zero before the voice service arrival rate is $450 \mathrm{call} / \mathrm{h}$, so the reserved channels can be used by the ordinary service. Figure 8 shows the ordinary voice service dropped call probability of PCR_QMB_DCA, QMB_DCA and MB_DCA algorithms. Before voice service arrival rate reaches 320 call/h the normal service of dropped call is zero in PCR_QMB_DCA which is later than it is in QMB_DCA, because the normal service of dropped call can use the reserved channels. When voice service 
arrival rate is higher than $440 \mathrm{call} / \mathrm{h}$, the ordinary voice service dropped call probability increases significantly in PCR_QMB_DCA. As shown in Figure 9, dropped call service probability of preemption priority service declines and delays, because the reserved channels can be used by preemption priority service with high priority. It can be concluded that reserving channel for non-emergency service can significantly improve its dropped call probability.

It can be seen from Figure 11 and 12 that when the arrival rate of voice and data service is at high level, the total utilizations of QMB_DCA algorithm channel and PCR_QMB_

DCA algorithm channel are 0.96 and 0.97 separately. Compare with QMB_DCA, PCR_QMB_DCA algorithm is improving 1\% approximately. However, when the arrival rate of voice service is at low level and data service is at high level, the total utilizations of QMB_DCA algorithm channel and PCR_QMB_DCA algorithm channel are 0.44 and 0.48 separately. Compare with QMB_DCA, PCR_QMB_DCA algorithm is improving 3\% approximately. The main reason is that, in PCR_QMB_DCA algorithm channel, when the arrival rate of voice is at low level, there is a greater probability of voice idle channel to be used by data service .In this way, the idle voice channel can be more effectively borrowed by data services.

\section{Conclusion}

The improved algorithms proposed based QMB_DCA algorithms in this paper, which reserved channel for dropped call, combine with grouping borrowing strategy and nonpatient customer. PCR_QMB_DCA algorithms have considered the different priority service and assigned different access conditions. From the simulation results, that the non-emergency voice service dropped call probability, the data blocking probability are improved, but the non-emergency voice service blocking probability is maintained, in a word, PCR_QMB_DCA algorithms improves the overall performances of the system and a better quality of service, to meet the different requirements of the business. The no patient customers introduced in this paper reflect the actual problems well and assist system optimization.

\section{Acknowledgments}

We gratefully thank the financial support by China Mobile group Xinjiang Co., Ltd (XJM2014-2788).

\section{References}

[1] C. H. Xiang and H. Lianfen, "Dynamic Channel Allocation Based On Genetic Algorithmic TD-SCDMA System”, IEEE, (2010), pp. 423-426.

[2] K. Chung, "Interference-adaptive fast dynamic channel allocation in TD-SCDMA system", Wireless Personal Communications, (2011), pp. 709-723.

[3] P. P. Li, J. Sun and S. Zhao, "A Fast Dynamic Channel Allocation Algorithm Based on Dynamic Area Division”, IEEE ICIME 3rd, (2011), pp. 361-364.

[4] S. Yi, L. Meng and T. Liangrui, "A Dynamic Channel Allocation Scheme Based On Handoff Reserving and New Call Queuing”, IEEE Computer Society, (2011), pp. 1-4.

[5] Z. Wang, P. T. Mathiopoulos and R. Chober, "Performance Analysis and Improvement Methods for Channel Resource Management Strategies of LEO-MSS with Multiparty Traffic”, IEEE Transactions on Vehicular Technology, vol. 57, no. 6, (2008), pp. 3832-3842.

[6] S. W. Xiao, Z. Y. Zhen and Z. Song, "Fast dynamic channel allocation scheme based on TD-SCDMA System", Journal of Jilin University (Engineering and Technology Edition), vol. 38, no. 4, (2008), pp. 955-959. 
[7] S. W. Xiao and Z. Jun, "TD-SCDMA Channel Allocation Technology based on switching channel reservation", Journal on Communications, vol. 30, no. 7, (2009), pp. 59-66.

[8] T. Hong and Z. Yan, "A Dynamic Channel Allocation Strategy Based on TD-SCDMA Trucking System", Chongqing University of Posts and Telecommunications Journal (Natural Sciences Edition), vol. 24, no. 1, (2012), pp. 29-33.

[9] “3GPP TSG UTRA TDD: Radio Transmission and Reception”, 3GPP TS 25.105, (2001).

[10] Z. Guo, J. Sun and S. Shao, "A Dynamic Channel Allocation Strategy Based on TD-SCDMA Trucking System", 7th International Conference on Wireless Communications, Networking and Mobile Computing, WiCOM, (2011).

[11] J. Zhen, "Fast dynamic channel allocation in TD-SCDMA system", Xi'an University of Electronic Science and Technology, (2013).

[12] C. Yanbo, C. Xingqing and L. Chengshu, "Dynamic Channel Allocation in TD-SCDMA", The 2003 IEEE International Conference on Communication Technology, (2003), pp. 1129-1132.

[13] S. Chengjun and Z. Zhongzhou, "Algorithm and Simulation for Fast DCA in TD-SCDMA", TENCON (2002), pp. 988-991.

\section{Authors}

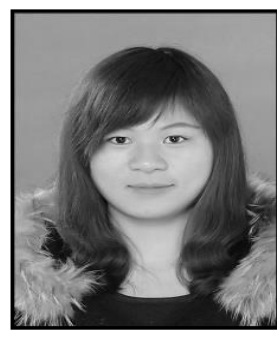

Hongyan Hu, she studied at the undergraduate College of Communication Engineering, Xinjiang University, and communication major. She is now a graduate student in Xinjiang University. Her research interests include singing and mobile Internet.

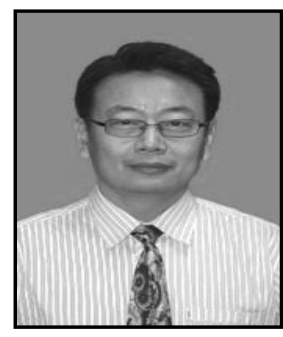

Zhenhong Jia, he received his BE from Beijing Normal University in1986. He received his $\mathrm{ME}$ and $\mathrm{PhD}$ from Shanghai Jiaotong University in 1989 and 1993. Currently, he is with the Xinjiang University where he is a Professor and a $\mathrm{PhD}$ tutor of information science. His research interests include optical communications, wireless communications. He has published over 100 papers. He was select for new century excellent talents plan in 2006. He is a member of Chinese Optical Society of Holography and Optical Information Processing Committee.

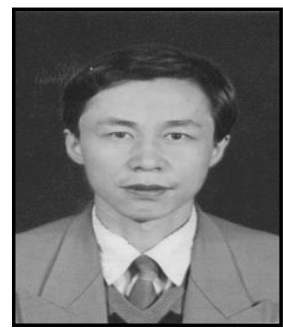

Xizhong Qin, he received his ME from Southeast University in1990. Currently, he is with the Xinjiang University where he is an associate Professor and a Master tutor of information science. His research interests include signal processing, wireless communications.

Chuanling Cao, she is an employee of China Mobile group Xinjiang Co., Ltd. Her major is service planning.

Hongmei Niu, she is an employee of China Mobile group Xinjiang Co., Ltd. Her specialty is network planning. 